\title{
Monojets and monophotons from light Higgsino pair production at LHC14
}

\author{
Howard Baer, ${ }^{1, *}$ Azar Mustafayev, ${ }^{2,3, \dagger}$ and Xerxes Tata ${ }^{2, \S}$ \\ ${ }^{1}$ Department of Physics and Astronomy, University of Oklahoma, Norman, Oklahoma 73019, USA \\ ${ }^{2}$ Department of Physics and Astronomy, University of Hawaii, Honolulu, Hawaii 96822, USA \\ ${ }^{3}$ Theory Division, CERN, CH-1211 Geneva 23, Switzerland
}

(Received 14 January 2014; published 11 March 2014)

\begin{abstract}
Naturalness arguments imply the existence of Higgsinos lighter than 200-300 GeV. However, because these Higgsinos are nearly mass degenerate, they release very little visible energy in their decays, and signals from electroweak Higgsino pair production typically remain buried under Standard Model backgrounds. Moreover, gluinos, squarks and winos may plausibly lie beyond the reach of the LHC14, so that signals from naturalness-inspired supersymmetric models may well remain hidden via conventional searches. We examine instead prospects for detecting Higgsino pair production via monojets or monophotons from initialstate radiation. We find typical signal-to-background rates at best at the $1 \%$ level and without any spectral distortions, leading to rather pessimistic conclusions regarding detectability via these channels.
\end{abstract}

\section{INTRODUCTION}

The minimization of the (renormalization-groupimproved one-loop) electroweak (EW) scalar potential of the minimal supersymmetric Standard Model (MSSM) leads to the well-known relation [1]

$$
\frac{M_{Z}^{2}}{2}=\frac{m_{H_{d}}^{2}+\Sigma_{d}^{d}-\left(m_{H_{u}}^{2}+\Sigma_{u}^{u}\right) \tan ^{2} \beta}{\tan ^{2} \beta-1}-\mu^{2}
$$

where the running potential parameters are evaluated at the scale $M_{\text {SUSY }}=\sqrt{m_{\tilde{t}_{1}} m_{\tilde{t}_{2}}}$ and where $\Sigma_{u}^{u}$ and $\Sigma_{d}^{d}$ are radiative corrections that arise from the derivatives of $\Delta V$ evaluated at the potential minimum. The sensitivity of $M_{Z}^{2}$ to the input parameters has been used to construct the necessary (though not sufficient) condition for naturalness defined by the electroweak fine-tuning measure [2,3],

$$
\Delta_{\mathrm{EW}} \equiv \max _{i}\left|C_{i}\right| /\left(M_{Z}^{2} / 2\right),
$$

where $\quad C_{H_{d}}=m_{H_{d}}^{2} /\left(\tan ^{2} \beta-1\right), \quad C_{H_{u}}=-m_{H_{u}}^{2} \tan ^{2} \beta /$ $\left(\tan ^{2} \beta-1\right)$ and $C_{\mu}=-\mu^{2}$. Also, $C_{\Sigma_{u}^{u}(k)}=-\Sigma_{u}^{u}(k) \tan ^{2} \beta /$ $\left(\tan ^{2} \beta-1\right)$ and $C_{\Sigma_{d}^{d}(k)}=\Sigma_{d}^{d}(k) /\left(\tan ^{2} \beta-1\right)$, where $k$ labels the various loop contributions included in Eq. (1). Expressions for the $\Sigma_{u}^{u}$ and $\Sigma_{d}^{d}$ are given in the Appendix of the second paper of Ref. [3].

Note that $\Delta_{\mathrm{EW}}$ is essentially determined by the supersymmetry (SUSY) spectrum. It is independent of both the underlying mechanism by which the superpartners acquire their masses and of the messenger scale, $\Lambda$, at which this mechanism is operative. This is in sharp contrast to conventional measures of fine-tuning such as $\Delta_{\mathrm{BG}}[4,5]$

\footnotetext{
*baer@nhn.ou.edu †azar@phys.hawaii.edu

\$tata@phys.hawaii.edu
}

or $\Delta_{\mathrm{HS}}[3,6,7]$ where corrections such as $\sim m_{H_{u}}^{2}(\Lambda) \ln \left(\frac{\Lambda^{2}}{m_{\text {scy }}^{2}}\right)$ lead to very high values of these fine-tuning measures especially in models - such as minimal supergravitywhere the parameters are defined at a very high energy scale. There is, of course, no contradiction since a small $\Delta_{\mathrm{EW}}$ is, as we have mentioned, just a necessary condition for fine-tuning $[3,8]$.

An immediate consequence of Eq. (1) is that models with values of $\mu^{2} \gg M_{Z}^{2}$ are necessarily fine-tuned. We emphasize that although we have used the electroweak scale minimization conditions to argue this, the same conclusion follows even with the use of popular fine-tuning measures. This is because $\mu^{2}$ runs very little between $M_{\mathrm{GUT}}$ and $M_{\text {SUSY }}$ so that the sensitivity of $M_{Z}^{2}$ to $\mu_{0}$, the grand unified theory (GUT) scale value of $\mu$, is changed by just $\sim 10 \%$. ${ }^{1}$ We thus conclude that a small value of $\mu^{2} /\left(M_{Z}^{2} / 2\right)$ is a robust and necessary condition for naturalness irrespective of the fine-tuning measure that is used. Stated differently, models with Higgsinos heavier than $200 \mathrm{GeV}(300 \mathrm{GeV})$ necessarily have a fine-tuning worse than $10 \%(3 \%)$. Experimental probes of light Higgsino pair production can thus decisively probe the naturalness of SUSY models. ${ }^{2}$

Motivated by these considerations, we have examined the spectra and aspects of the phenomenology that result in models where $\Delta_{\mathrm{EW}} \sim 10-30$. Typically, the dominant radiative corrections to Eq. (1) come from the top-squark contributions $\Sigma_{u}^{u}\left(\tilde{t}_{1,2}\right)$. For negative values of the trilinear

\footnotetext{
${ }^{1}$ This simple fact often remains obscured because the values of both $\Delta_{\mathrm{HS}}$ and $\Delta_{\mathrm{BG}}$ are defined by the input parameter that $M_{Z}^{2}$ is most sensitive to, and this is almost never $\mu_{0}^{2}$.

${ }^{2}$ Note that the link between fine-tuning and the Higgsino mass breaks down if the dominant contribution to the Higgsino mass is nonsupersymmetric [9]. If there are no singlets that couple to Higgsinos, such a contribution would be soft. However, in all high-scale models that we know, the Higgsino mass has a supersymmetric origin.
} 
soft term $A_{t}$ somewhat larger than the GUT scale scalar masses, each of $\Sigma_{u}^{u}\left(\tilde{t}_{1}\right)$ and $\Sigma_{u}^{u}\left(\tilde{t}_{2}\right)$ can be minimized whilst lifting up $m_{h}$ into the $125 \mathrm{GeV}$ regime [2] as required by the discovery of the Higgs boson at the LHC [10,11]. Upon requiring no large independent contributions to Eq. (1) (which would necessitate fine-tuning of the remaining parameters to keep $M_{Z}$ at $\simeq 91.2 \mathrm{GeV}$ ), we find that

(i) $|\mu| \sim 100-300 \mathrm{GeV}$ (the closer to $M_{Z}$ the better),

(ii) $m_{H_{u}}^{2}$ is driven radiatively to only small negative values,

(iii) the top squarks which enter the $\Sigma_{u}^{u}$ radiative corrections are highly mixed and lie at or around the few $\mathrm{TeV}$ scale and

(iv) in order to keep $m_{\tilde{t}_{1,2}}$ from growing too large, the gluino mass is also bounded from above, in this case by $m_{\tilde{g}} \lesssim 4-5 \mathrm{TeV}$.

Sparticle mass spectra consistent with a low $\Delta_{\mathrm{EW}}$ can readily yield a value of $m_{h} \sim 125 \mathrm{GeV}$ whilst evading LHC8 search limits on squarks, gluinos and top squarks $[12,13]$, and at the same time maintaining low electroweak fine-tuning, our necessary condition for naturalness. The key feature of the mass spectra implied by Eq. (1) is the existence of four light Higgsinos- $\tilde{W}_{1}^{ \pm}, \tilde{Z}_{1}$ and $\tilde{Z}_{2}$-all with mass $\sim|\mu| \sim 100-300 \mathrm{GeV}$. While these light Higgsinos can be produced at the LHC at large rates, their compressed spectra with mass gaps $m_{\tilde{Z}_{2}}-m_{\tilde{Z}_{1}} \sim m_{\tilde{W}_{1}}-m_{\tilde{Z}_{1}} \sim$ $10-30 \mathrm{GeV}$ results in only soft visible energy release from their three-body decays; this makes signal extraction from the SM background exceedingly difficult, if not impossible.

A new signature endemic to models with light Higgsinos has also been pointed out in Ref. [14]: $p p \rightarrow \tilde{W}_{2}^{ \pm} \tilde{Z}_{4} \rightarrow$ $\left(\tilde{Z}_{1,2} W^{ \pm}\right)+\left(\tilde{W}_{1}^{\mp} W^{ \pm}\right)$which results in hadronically quiet -because the decay products of $\tilde{W}_{1}$ and $\tilde{Z}_{2}$ are softsame-sign diboson events (SSdB). The $300 \mathrm{fb}^{-1}$ LHC14 reach for SSdBs extends to a wino mass of about $700 \mathrm{GeV}$. This corresponds to $m_{\tilde{g}} \sim 2.1 \mathrm{TeV}$ in models with gaugino mass unification, somewhat larger than the LHC14 reach for gluino pair production [15]. Confirmatory signals will also be present in multilepton channels [15]. Since $m_{\tilde{g}}$ can extend up to $4-5 \mathrm{TeV}$ while maintaining low $\Delta_{\mathrm{EW}} \lesssim 30$, then LHC14 can probe only a fraction of the parameter space of natural SUSY in this manner.

An alternative LHC search strategy has been proposed in a variety of papers (for a summary and detailed references, see e.g. Ref. [16]), namely to look for initial-state QED/QCD radiation off weakly interacting massive particle (WIMP) pair production. Much of this work [17-23] has been carried out using effective operator analyses. Here, it is assumed that the interactions between the dark matter particle and SM quarks occur via very heavy mediators (usually $t$ - and $u$-channel squarks in the context of the MSSM bino-like WIMP) so that the contact approximation is valid. It is clear that for MSSM Higgsino pair production the contact interaction approximation breaks down very badly because Higgsinos are dominantly produced by collisions of quarks and antiquarks (inside the protons) via $s$-channel $Z$ exchange. Since Higgsinos are necessarily heavier than $\sim 100 \mathrm{GeV}$, the $Z$-boson propagator suppresses the amplitude for Higgsino production by an extra factor of $\hat{s}$ relative to the contactinteraction approximation. This results in a suppression of the cross section where the Higgsino pair is produced with large invariant mass. Since the radiation of hard gluons or photons is most likely in this regime, the contact-interaction approximation will badly overestimate the cross section for high- $E_{T}$ monojet and monophoton events, as has already been emphasized in Ref. [24]. As a result, constraints [22,23] using effective operator analyses, therefore, do not apply in the case that the light SUSY states are Higgsinos.

In a recent analysis, Han et al. [25] have computed the monojet signal in the natural SUSY framework with light Higgsinos using the complete matrix element. An advantage of applying this technique to models with light Higgsinos is that one is not restricted to just WIMP $\left(\tilde{Z}_{1}\right)$ pair production, but one may radiate off gluons or photons in several other reactions as well: $p p \rightarrow \tilde{W}_{1}^{+} \tilde{W}_{1}^{-}, \tilde{Z}_{1} \tilde{Z}_{2}$, $\tilde{Z}_{2} \tilde{Z}_{1}$ and $\tilde{W}_{1} \tilde{Z}_{1,2}$, since again the heavier Higgsino decay debris is expected to be soft (unless highly boosted) at the LHC. Including all the relevant contributions, these authors claim that LHC14, with an integrated luminosity of $1500 \mathrm{fb}^{-1}$ will be able to probe Higgsinos up to $200 \mathrm{GeV}$ at $5 \sigma$ [25]. If their results hold up to scrutiny, it will imply that experiments at the high-luminosity upgrade of the LHC will decisively probe SUSY models fine-tuned to no more than $10 \%$. $^{3}$

Given the importance of this result, we reexamine prospects for the detection of monojet radiation off of Higgsino pair production in Sec. II. Our conclusions are, however, quite different from those of Ref. [25] since we find signal well below SM backgrounds (at the percent level), with no distinctive monojet features which would allow for the separation of signal from background. In Sec. III, we perform similar calculations for monophoton radiation and arrive at similarly pessimistic conclusions. We have decided such a pessimistic assessment is worthy of publication not only because of the optimistic claims in the literature [25], but also to highlight that claims about the observability of monojet/monophoton signals from effective operator analyses should be viewed with caution.

\section{PROSPECTS FOR MONOJETS}

To examine signal rates, we first select a low- $\Delta_{\mathrm{EW}}$ SUSY benchmark model from radiatively driven natural SUSY

\footnotetext{
${ }^{3}$ After our paper was submitted, the authors of Ref. [25] put out a revised analysis, in which they modified their treatment of the error on the backgrounds (see Footnote 4 below). Their latest analysis (Ref. [25]) claims a $3 \sigma(5 \sigma)$ signal for $|\mu|=160$ (110) $\mathrm{GeV}$ at LHC14 with $3000 \mathrm{fb}^{-1}$. We retain mention of their earlier results in the text to provide the reader with proper perspective for our analysis.
} 
(RNS) which uses the two-extra-parameter nonuniversal Higgs model (NUHM2) with input parameters

$$
m_{0}, \quad m_{1 / 2}, \quad A_{0}, \quad \tan \beta, \quad \mu, \quad m_{A},
$$

with $m_{t}=173.2 \mathrm{GeV}$. We generate the sparticle spectrum using ISAJET 7.84 [26]. We fix $m_{0}=5 \mathrm{TeV}, m_{1 / 2}=$ $750 \mathrm{GeV}, A_{0}=-8 \mathrm{TeV}, \tan \beta=10, \mu=150 \mathrm{GeV}$ and $m_{A}=1 \mathrm{TeV}$. This leads to a sparticle spectrum with $m_{\tilde{g}}=1.9 \mathrm{TeV}$, very heavy squarks and sleptons, binos and winos with masses of several hundred $\mathrm{GeV}$, and a set of Higgsinos with $m_{\tilde{W}_{1}^{ \pm}}=155.6 \mathrm{GeV}, m_{\tilde{Z}_{2}}=158.9 \mathrm{GeV}$ and $m_{\tilde{Z}_{1}}=142.2 \mathrm{GeV}$. These Higgsinos are, of course, the focus of the present study, and our broad conclusions are essentially independent of the rest of the spectrum, as long as the bino and wino states are much heavier than the Higgsino states. The value of $\Delta_{\mathrm{EW}}=19.7$.

We use MADGRAPH 5 [27] to generate $p p \rightarrow \tilde{W}_{1}^{+} \tilde{W}_{1}^{-}$, $\tilde{Z}_{1,2} \tilde{Z}_{1,2}$ and $\tilde{W}_{1}^{ \pm} \tilde{Z}_{1,2}$ plus one-parton processes (exclusive) and plus two-parton processes (inclusive) where for efficiency we require the hardest final-state parton to have $p_{T}$ (parton) $>120 \mathrm{GeV}$; the final cross section is then the sum of one-jet exclusive and two-jet inclusive processes. We also evaluate the $Z+$ jets, $W+$ jets and $Z Z+$ jets backgrounds (where $Z$ 's decay to neutrinos and $W$ 's decay leptonically) in a similar fashion, as the sum of one- and two-parton processes. To avoid double counting, we used the MLM scheme for jet-parton matching [28]. The events are then passed to PYTHIA [29] for showering, hadronization and underlying events. We have not evaluated the hard monojet background from top-quark pair production which we expect to be very small after the veto on additional jets and leptons. This is confirmed by the results of Ref. [25].

The MADGRAPH/PYTHIA events are then passed to the ISAJET toy detector simulation with calorimeter cell size $\Delta \eta \times \Delta \phi=0.05 \times 0.05$ and $-5<\eta<5$. The hadronic calorimetry energy resolution is taken to be $80 \% / \sqrt{E}+$ $3 \%$ for $|\eta|<2.6$ and the forward calorimetry is $100 \% / \sqrt{E}+5 \%$ for $|\eta|>2.6$, where the two terms are combined in quadrature. The electromagnetic calorimetry energy resolution is assumed to be $3 \% / \sqrt{E}+0.5 \%$. We use the cone-type ISAJET jet-finding algorithm [26] to group the hadronic final states into jets. Jets and isolated leptons are defined as follows:

(i) Jets are hadronic clusters with $|\eta|<3.0, R \equiv$ $\sqrt{\Delta \eta^{2}+\Delta \phi^{2}} \leq 0.4$ and $E_{T}($ jet $)>40 \mathrm{GeV}$.

(ii) Electrons and muons are considered isolated if they have $|\eta|<2.5, p_{T}(l)>10 \mathrm{GeV}$ with visible activity within a cone of $\Delta R<0.2$ about the lepton direction, $\Sigma E_{T}^{\text {cells }}<5 \mathrm{GeV}$.

(iii) Jets with just one or three charged particles are labeled as taus.

(iv) We identify hadronic clusters as $b$-jets if they contain a $B$ hadron with $E_{T}(B)>15 \mathrm{GeV}, \eta(B)<3$ and $\Delta R(B$, jet $)<0.5$. We assume a tagging efficiency of $60 \%$ and light-quark and gluon jets can be mistagged as a $b$-jet with a probability $1 / 150$ for $E_{T} \leq 100 \mathrm{GeV}$, and $1 / 50$ for $E_{T} \geq 250 \mathrm{GeV}$, with a linear interpolation for intermediate $E_{T}$ values.

Following the ATLAS monojet study [30], we impose the following cuts:

(i) $n$ (jets) $\leq 2$ for $p_{T}$ (jet) $>30 \mathrm{GeV}$;

(ii) if $n$ (jets) $=2$, then $p_{T}\left(j_{2}\right)<100 \mathrm{GeV}$;

(iii) $b$-jet veto ( $b$-jets as defined above), to eliminate top backgrounds;

(iv) $\tau$-jet veto;

(v) isolated lepton veto.

Our resulting distributions are shown in Fig. 1 for (a) $p_{T}\left(j_{1}\right)$ and (b) $E_{T}^{\text {miss }}$. As expected, the shapes of the two distributions are similar for large $p_{T}(j)$ and large $E_{T}^{\text {miss }}$ but begin to differ for values below $\sim 200 \mathrm{GeV}$ where details of event generation and the presence of the second jet may be important. From Fig. 1(a), we see that $Z+$ jets production forms the dominant background, followed closely by $W+$ jets production where the lepton from $W$ decay is too soft or buried within a jet or too forward or
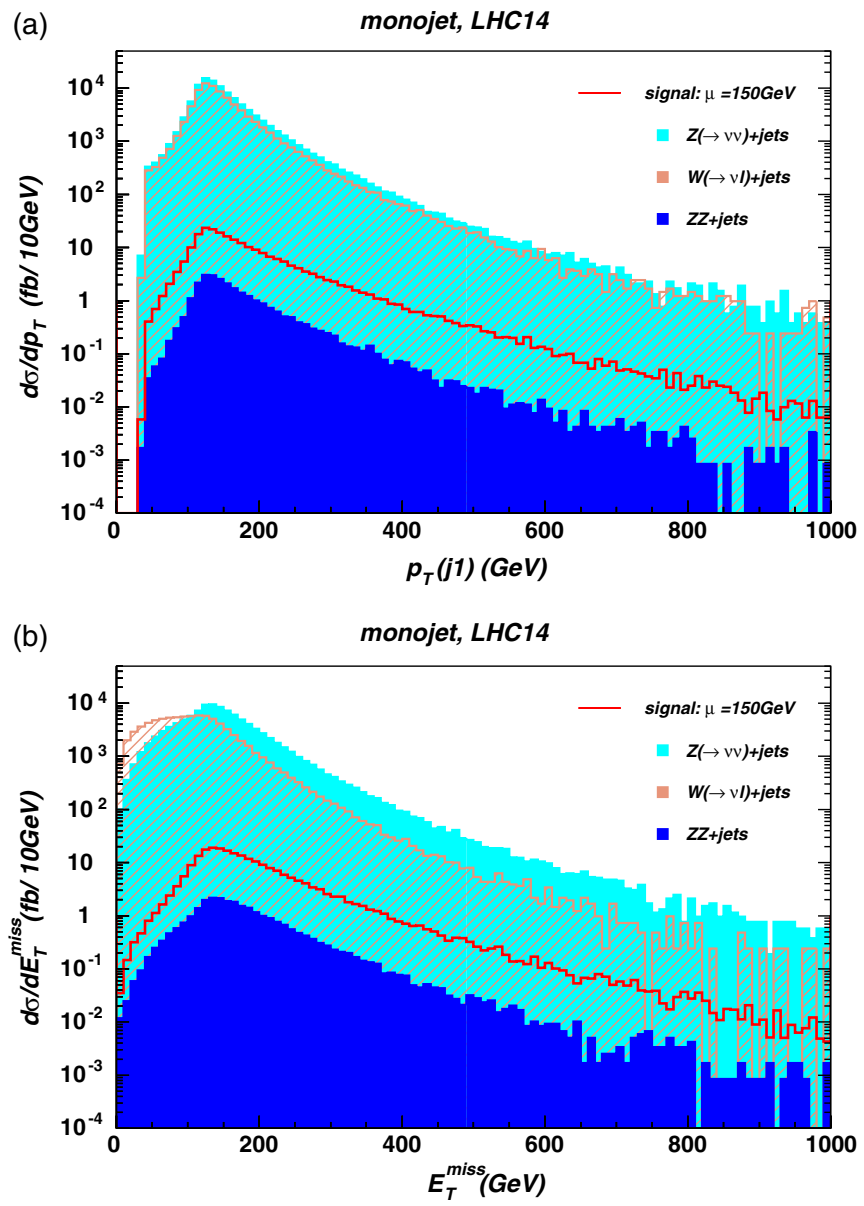

FIG. 1 (color online). Distribution in (a) $p_{T}$ (jet) and (b) $E_{T}^{\text {miss }}$ from initial-state radiation off Higgsino pair production at LHC14. We also show backgrounds from $Z+$ jets, $W+$ jets and $Z Z+$ jets production, where $W \rightarrow \ell \nu$ and $Z \rightarrow \nu \bar{\nu}$. 
TABLE I. Cut flow for the dominant backgrounds in the monojet channel and for the RNS signal with $\mu=150 \mathrm{GeV}$. Both $Z$ 's in column 4 are forced to decay to neutrinos. All cross sections are in fb.

\begin{tabular}{|c|c|c|c|c|}
\hline Before cuts & $\begin{array}{c}Z(\nu \bar{\nu})+\text { jets } \\
146740\end{array}$ & $\begin{array}{l}W(l \nu)+\text { jets } \\
488282\end{array}$ & $\begin{array}{c}Z Z+\text { jets } \\
37.747\end{array}$ & $\begin{array}{c}\text { Signal } \\
349.717\end{array}$ \\
\hline$n($ jets $) \geq 1$ with $\left|\eta\left(j_{1}\right)\right|<2.0$ & 118814 & 408716 & 33.041 & 304.251 \\
\hline$p_{T}\left(j_{1}\right)>500 \mathrm{GeV}$ & 816.87 & 3078.23 & 0.760 & 9.913 \\
\hline$E_{T}^{\text {miss }}>500 \mathrm{GeV}$ & 319.83 & 380.16 & 0.370 & 6.611 \\
\hline$\Delta \phi\left(j_{2}, E_{T}^{\mathrm{miss}}\right)>0.5$ & 296.54 & 300.43 & 0.333 & 5.351 \\
\hline veto on $p_{T}\left(j_{3}\right)>30 \mathrm{GeV},\left|\eta\left(j_{3}\right)\right|<4.5$ & 249.38 & 215.36 & 0.273 & 3.544 \\
\hline veto on $e, \mu$ & 249.38 & 45.70 & 0.273 & 2.885 \\
\hline veto on $\tau$-jets & 247.85 & 45.21 & 0.270 & 2.867 \\
\hline veto on $b$-jets & 241.93 & 44.72 & 0.267 & 2.799 \\
\hline
\end{tabular}

otherwise unidentified. The signal is shown by the red solid histogram and lies typically about two orders of magnitude below the background distribution. We also show the distribution from $Z Z+$ jets production, which is subdominant. Essentially the same qualitative features are also seen in Fig. 1(b). Nowhere in either panel does the signal emerge from background. Other cuts such as angular distributions do not help the situation since both signal and background

(a)

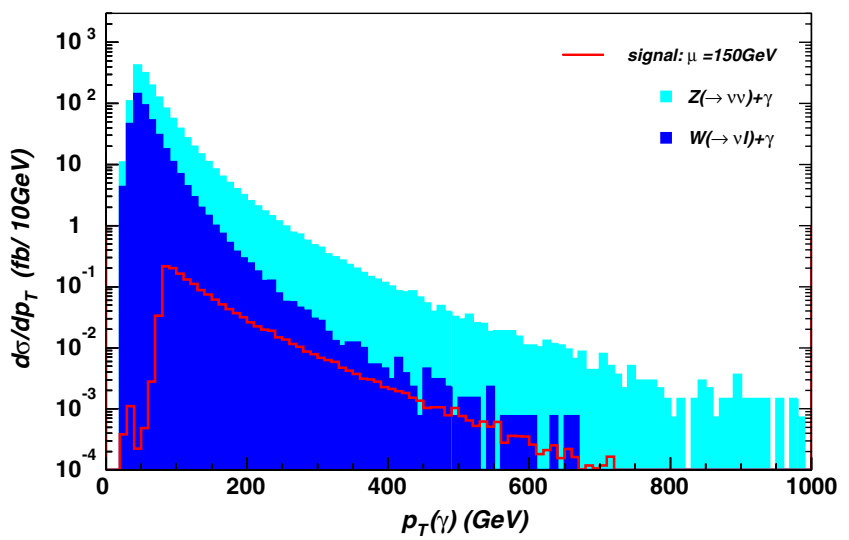

(b)

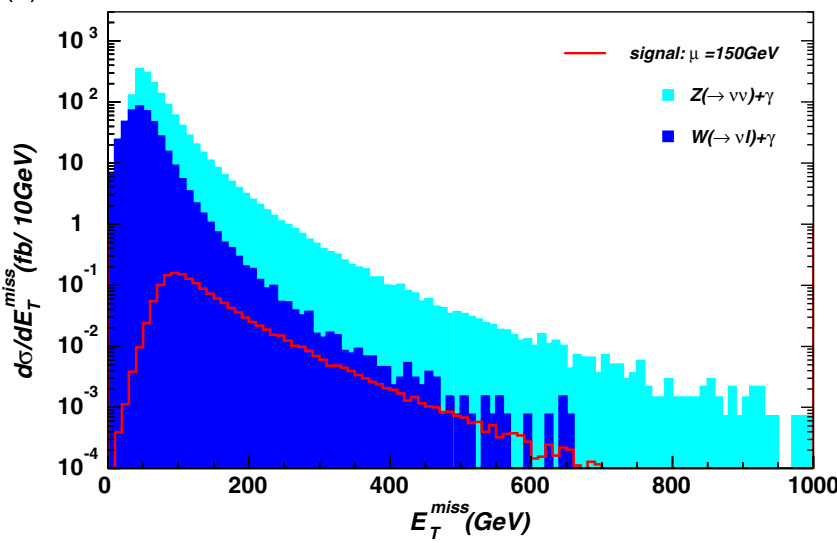

FIG. 2 (color online). Distribution in (a) $p_{T}(\gamma)$ and (b) $E_{T}^{\text {miss }}$ from initial-state photon radiation off Higgsino pair production at LHC14. We also show backgrounds from $Z \gamma$ and $W \gamma$ production. are dominated by gluon radiation off initial-state quarks: really, the main difference between signal and background as far as initial state radiation (ISR) goes is that for signal the ISR comes off a somewhat higher $Q^{2}$ subprocess. The effect of sequential cuts on the signal and on the background is shown in Table I.

Given that the signal and background have similar shapes and that $S / B \sim 1 \%$, it is very difficult to make the case that it will be possible to realistically extract the signal [31]. Of course, with sufficient integrated luminosity, the statistical significance will always exceed $5 \sigma$, but to claim that this means the signal is observable means that the background is known with a precision better than a percent! ${ }^{4}$

\section{PROSPECTS FOR MONOPHOTONS}

For monophoton events (which we include for completeness), we generate the same signal sample as before,

\footnotetext{
${ }^{4}$ We have traditionally included the requirement of $S / B>0.2$ in addition to the $5 \sigma$ statistical significance level and to a minimum 5-10 event level for the observability of the signal. With this criterion, the signal is clearly unobservable. That the $Z(\rightarrow \nu \nu)+$ jet background may be directly inferred from the observed $Z(\rightarrow \ell \bar{\ell})+$ jet events does not change the situation because the statistical fluctuations of this background remain too large except for very high integrated luminosities. Moreover, the theoretical systematic from the $W j$ background still swamps the signal. We note that up to factors of about 1.5-2, our signal and background rates are compatible with those in Table I of Ref. [25]: i.e. we are in qualitative agreement with their calculation of the cross sections. In their analysis, Han et al. attributed a scaled statistical error to the $Z(\rightarrow \nu \bar{\nu})+j$ background but neglected any systematic error on this background which can be extracted from data, and included a statistical as well as a $10 \%$ theoretical uncertainty in the other backgrounds. To get the total uncertainty, they then combined the statistical and theoretical errors in quadrature. For integrated luminosities of $\mathcal{O}(1000) \mathrm{fb}^{-1}$ that these authors found necessary to claim a signal, the systematic error (which will not improve with integrated luminosity), will completely dominate the statistical error unless one assumes that the systematic uncertainties can be reduced to about the percent level, something we regard to be unrealistic. Once the systematic error is properly incorporated, Han et al. agree with us that the signal is unobservable in the monojet channel [32].
} 
TABLE II. Cut flow for the dominant backgrounds in the monophoton channel and for the RNS signal with $\mu=150 \mathrm{GeV}$. All cross sections are in $\mathrm{fb}$.

\begin{tabular}{|c|c|c|c|}
\hline Before cuts & $\begin{array}{c}Z(\nu \bar{\nu})+\gamma \\
1826.77\end{array}$ & $\begin{array}{c}W(l \nu)+\gamma \\
2296.76\end{array}$ & $\begin{array}{r}\text { Signal } \\
3.083 \\
\end{array}$ \\
\hline$n($ photon $) \geq 1$ & 1756.92 & 2205.32 & 2.895 \\
\hline$p_{T}(\gamma 1)>150 \mathrm{GeV}$ & 63.79 & 75.58 & 0.997 \\
\hline$E_{T}^{\text {miss }}>150 \mathrm{GeV}$ & 49.43 & 15.42 & 0.778 \\
\hline$n($ jets $) \leq 1, \mid \eta($ jet $) \mid<4.5$ & 39.60 & 9.43 & 0.473 \\
\hline veto on $e, \mu$ & 39.60 & 2.67 & 0.418 \\
\hline veto on $\tau$-jets & 36.15 & 2.45 & 0.371 \\
\hline
\end{tabular}

including all Higgsino pair production reactions, but now requiring one hard photon (with $p_{T}>40 \mathrm{GeV}$ ) radiation instead of a hard jet. We also generate the background production processes $Z \gamma$ (followed by $Z \rightarrow \nu \bar{\nu}$ ) and $W \gamma$ (followed by $W \rightarrow \ell \nu_{\ell}$ where $\ell=e, \mu$ or $\tau$ ) as before using MADGRAPH plus PYTHIA.

For the isolated monophoton sample, we require [23]

(i) $n(\gamma) \geq 1$,

(ii) $n$ (jets) $\leq 1$ with $\mid \eta$ (jet) $\mid<4.5$,

(iii) tau-jet veto, and

(iv) isolated lepton veto.

We regard a photon to be isolated if the energy in a cone of radius $\Delta R<0.4$ around a photon with $p_{T}(\gamma)>25 \mathrm{GeV}$, $|\eta(\gamma)|<2.5$ is less than $5 \mathrm{GeV}$.

Our signal and background distributions in $p_{T}(\gamma)$ and $E_{T}^{\text {miss }}$ are shown in Fig. 2. As in Fig. 1, we see that the shapes agree for large values of $p_{T}(\gamma)$ and $E_{T}^{\text {miss }}$. For the entire range of $p_{T}(\gamma)$ as well as of $E_{T}^{\text {miss }}$, we again find that signal (solid red histogram) lies below the $Z \gamma$ background by typically two orders of magnitude. The $W \gamma$ background falls more sharply than the $Z \gamma$ background. This is because when we require much higher $p_{T}(\gamma)$ values, then the $W$ recoils more sharply against the gamma, and its decay products are more likely to be hard and isolated, and to not pass the lepton/tau veto requirements. The effect of the sequential cuts on the signal and background cross sections is shown in Table II. Once again, there are no distinctive features in the distribution, and as for the monojet signal of the previous section, we deem the monophoton signal to be unobservable because of the very small $S / B$ ratio.

\section{CONCLUDING REMARKS}

The existence of light Higgsinos with masses smaller than 200-300 GeV (depending on how much fine-tuning one is willing to tolerate) is a robust feature of natural SUSY models. Although these Higgsinos can be pair produced at large rates at the LHC, the signal will be buried below SM backgrounds because of the small energy release from their decays. In this paper, we have examined prospects for their detection via pair production in association with a hard jet or a hard, isolated photon resulting in characteristic monojet or monophoton events at LHC14. We emphasize here that constraints obtained from analyses $[22,23]$ using contact interactions between quarks and the Higgsinos are inapplicable in this connection because the effective-operator approximation fails badly for Higgsino pair production.

While monojet and monophoton signal events indeed occur at an observable rate particularly at the luminosity upgrade of the LHC, we are pessimistic about the prospects for their detection because backgrounds from $Z$ and $W$ production in association with a jet or an isolated photon overwhelm the signal by two orders of magnitude even for very large values of jet or photon transverse momentum and $E_{T}^{\text {miss }}$ in these events. It seems to us difficult to imagine that it would be possible to claim a signal for new physics in these channels based solely on an excess of $\mathcal{O}(1 \%)$ without an observable distortion of any distribution.

In arriving at our negative conclusion, we should mention that we have not investigated whether it might be possible to extract the Higgsino signal by examining the soft debris from the decays of $\tilde{W}_{1}$ and $\tilde{Z}_{2}$ produced via $\tilde{W}_{1} \tilde{Z}_{2}, \tilde{Z}_{1} \tilde{Z}_{2}$ and $\tilde{W}_{1} \tilde{W}_{1}$ pair production processes that dominate Higgsino pair production [15]. This will require a careful analysis of potential backgrounds from higher-order Standard Model processes. Despite our cautious pessimism, we leave open the possibility that a clever analysis may make it feasible to tease out this signal at a luminosity upgrade of LHC14.

\section{ACKNOWLEDGMENTS}

A. M. thanks CERN Theory Division for its support and hospitality. This work was supported in part by the US Department of Energy, Office of High Energy Physics.

Note added.-After this study was completed we saw Ref. [33] which claims that exclusion (not discovery) of electroweak-ino masses up to $200 \mathrm{GeV}$ is possible with $300 \mathrm{fb}^{-1}$ of integrated luminosity at LHC14 if the systematic error can be reduced to the $1 \%$ level. 
[1] See, e.g. H. Baer and X. Tata, Weak Scale Supersymmetry: From Superfields to Scattering Events, (Cambridge University Press, Cambridge, 2006).

[2] H. Baer, V. Barger, P. Huang, A. Mustafayev, and X. Tata, Phys. Rev. Lett. 109, 161802 (2012).

[3] H. Baer, V. Barger, P. Huang, D. Mickelson, A. Mustafayev, and X. Tata, Phys. Rev. D 87, 035017 (2013); 87, 115028 (2013).

[4] J. Ellis, K. Enqvist, D. Nanopoulos, and F. Zwirner, Mod. Phys. Lett. A 01, 57 (1986).

[5] R. Barbieri and G. Guidice, Nucl. Phys. B306, 36 (1988).

[6] R. Kitano and Y. Nomura, Phys. Lett. B 631, 58 (2005); Phys. Rev. D 73, 095004 (2006); , Report No. SLAC-PUB11892.

[7] M. Papucci, J. T. Ruderman, and A. Weiler, J. High Energy Phys. 09 (2012) 035.

[8] H. Baer, V. Barger, and D. Mickelson, Phys. Rev. D 88, 095013 (2013).

[9] C. Brust, A. Katz, S. Lawrence, and R. Sundrum, J. High Energy Phys. 03 (2012) 103.

[10] G. Aad et al. (ATLAS Collaboration), Phys. Lett. B 716, 1 (2012).

[11] S. Chatrchyan et al. (CMS Collaboration), Phys. Lett. B 716, 30 (2012).

[12] G. Aad et al. (ATLAS Collaboration), Phys. Rev. D 87, 012008 (2013); (ATLAS Collaboration), Report No. ATLAS-CONF-2013-04; (ATLAS Collaboration), Report No. ATLAS-CONF-2013-061.

[13] S. Chatrchyan et al. (CMS Collaboration), J. High Energy Phys. 10 (2012) 018; (CMS Collaboration), Report No. CMS-PAS-SUS-13-007.

[14] H. Baer, V. Barger, P. Huang, D. Mickelson, A. Mustafayev, W. Sreethawong, and X. Tata, Phys. Rev. Lett. 110, 151801 (2013).

[15] H. Baer, V. Barger, P. Huang, D. Mickelson, A. Mustafayev, W. Sreethawong, and X. Tata, J. High Energy Phys. 12 (2013) 013.
[16] S. Arrenberg et al., arXiv:1310.8621.

[17] H. Zhang, Q. Cao, C. Chen, and C. Li, J. High Energy Phys. 08 (2011) 018.

[18] M. Beltrán, D. Hooper, E. W. Kolb, Z. A. C. Krusberg, and T. M. P. Tait, J. High Energy Phys. 09 (2010) 037.

[19] J. Goodman, M. Ibe, A. Rajaraman, W. Shepherd, T. M. P. Tait, and H.-B. Yu, Phys. Rev. D 82, 116010 (2010).

[20] A. Rajaraman, W. Shepherd, T. Tait, and A. Wijangco, Phys. Rev. D 84, 095013 (2011).

[21] P. Fox, R. Harnik, and J. Kopp, Phys. Rev. D 85, 056011 (2012).

[22] S. Chatrchyan et al. (CMS Collaboration), J. High Energy Phys. 09 (2012) 094; (CMS Collaboration), Phys. Rev. Lett. 108, 261803 (2012).

[23] G. Aad et al. (ATLAS Collaboration), J. High Energy Phys. 04 (2013) 075; (ATLAS Collaboration), Phys. Rev. Lett. 110, 011802 (2013).

[24] O. Buchmueller, M. Dolan, and C. McCabe, J. High Energy Phys. 01 (2014) 025.

[25] C. Han, A. Kobakhidze, N. Liu, A. Saavedra, L. Wu, and J. M. Yang, J. High Energy Phys. (2014) 049.

[26] ISAJET, by H. Baer, F. Paige, S. Protopopescu, and X. Tata, arXiv:hep-ph/0312045.

[27] J. Alwall, M. Herquet, F. Maltoni, O. Mattelaer, and T. Stelzer, J. High Energy Phys. 06 (2011) 128.

[28] M. Mangano, M. Moretti, F. Piccinini, and M. Treccani, J. High Energy Phys. 01 (2007) 013.

[29] T. Sjostrand, S. Mrenna, and P. Z. Skands, J. High Energy Phys. 05 (2006) 026.

[30] ATLAS Collaboration, Report No. ATLAS-CONF-2011096.

[31] G. Giudice, T. Han, K. Wang, and L.-T. Wang, Phys. Rev. D 81, 115011 (2010).

[32] L. Wu and J. M. Yang (private communication).

[33] P. Schwaller and J. Zurita, arXiv:1312.7350. 